Blood Cells Mol Dis. 2010 December 15; 45(4): 326-333. doi:10.1016/j.bcmd.2010.10.002.

\title{
Zebrafish von Willebrand Factor
}

\author{
Maira Carrillo, Seongcheol Kim, Surendra Kumar Rajpurohit, Vrinda Kulkarni, and Pudur \\ Jagadeeswaran \\ Department of Biological Sciences University of North Texas, Denton, TX 76203
}

\section{Abstract}

von Willebrand factor (vWF) is a large protein involved in primary hemostasis. A dysfunction in this protein or an insufficient production of the protein leads to improper platelet adhesion/ aggregation, resulting in a bleeding phenotype known as von Willebrand disease (vWD). To gain a better understanding of vWF interactions in vivo, the use of zebrafish as a model is ideal because of the transparency of the embryos and larvae. In this article, we examined the presence and function of vWF in hemostasis of zebrafish utilizing a variety of molecular methods. Using RTPCR and antibody staining, we have shown that vWF mRNA is present in thrombocytes. Through antibody staining, we demonstrated vWF is synthesized in blood vessels. The role of zebrafish vWF in hemostasis was established through knockdown methods using vWF morpholino (vWF MO) antisense oligonucleotides. Embryos injected with vWF MO at the one to four cell stages resulted in a bleeding phenotype. Injection of embryos with vWF MO also caused an increase in time to occlusion within arteries in larvae upon laser induced injury. We then used vWF-specific Vivo-Morpholinos (VMO) to induce vWF knockdown in adult zebrafish by targeting the exon homologous to the human exon 28 of the vWF gene. The reduced ristocetin-mediated agglutination of thrombocytes in a plate tilting assay, using blood from adult zebrafish injected with VMO, provided evidence that $\mathrm{vWF}$ is involved in the hemostatic process. We also administered desmopressin acetate to larvae and adults which resulted in enhanced aggregation/ agglutination of thrombocytes. Zebrafish genome database analysis revealed the presence of GPIb $\beta$ gene. It also revealed the exon of zebrafish vWF gene corresponding to exon 28 of human vWF gen is highly similar to the exon 28 of human vWF gene, except that it has an insertion that leads to a translated peptide sequence that separates the two A domains coded by this exon. This exon is also conserved in other fishes. In summary, we established that zebrafish vWF has a role similar to that of vWF found in humans, thus, making zebrafish a useful model for studying the cell biology of vWF in vivo.

\section{Keywords}

zebrafish; von Willebrand factor; thrombocytes; thrombosis; GPIb $\beta$

\section{Introduction}

von Willebrand factor (vWF) plays an integral role in primary hemostasis. In humans, von Willebrand factor is a large protein of 2813 amino acids encoded by the vWF gene, which is

(C) 2010 Elsevier Inc. All rights reserved.

Corresponding author: Pudur Jagadeeswaran Phone: 940-595-2736 Fax: 940-565-3821 Jag@unt.edu.

Publisher's Disclaimer: This is a PDF file of an unedited manuscript that has been accepted for publication. As a service to our customers we are providing this early version of the manuscript. The manuscript will undergo copyediting, typesetting, and review of the resulting proof before it is published in its final citable form. Please note that during the production process errors may be discovered which could affect the content, and all legal disclaimers that apply to the journal pertain. 
composed of 52 exons and is located on chromosome 12 [1]. The 2813 amino acid sequence includes a signal peptide of 22 residues, a propeptide of 741 residues, and a mature subunit of 2050 amino acids [1]. Synthesis of vWF occurs in endothelial cells as well as in megakaryocytes; vWF is stored in the Weibel-Palade bodies of endothelial cells and the $\alpha$ granules of platelets, which are derived from megakaryocytes [2]. The role of vWF is to stabilize factor VIII, to act as an adhesive by binding to the subendothelium at the site of injury, and to promote platelet adhesion/aggregation via binding to the platelet receptor GpIb $\alpha$ [2]. The ability of vWF to form a stable hemostatic plug is imperative in arteries, due to the rates of blood flow within the vessels [3]. Evidence of the importance of vWF is apparent in individuals suffering from von Willebrand disease (vWD). vWD manifests as a qualitative or quantitative dysfunction, and is characterized by mucocutaneous bleeding and prolonged clotting time. The majority of the mutations in the vWF gene causing vWD is clustered within two A domains encoded by exon 28 .

The study of vWD is important due to the fact that $1 \%$ of the population is afflicted with the disease [4]. To effectively study vWF and vWD, the employment of animal models is essential. Currently the models in use include murine, canine, rabbit and porcine, all of which have provided valuable information about vWF and vWD [5;6]. However, these models all present a challenge in that it is difficult to image vessel occlusion in vivo or to induce injury without being highly invasive. Additionally, observation of developing offspring is difficult due to the fact that all of these models develop in utero. Currently, another animal model, the zebrafish Danio rerio, is being used for studying hemostasis, but is not yet employed as a tool to study vWF and vWD [7;8]. Zebrafish have previously been shown to retain many of the clotting factors involved in both the intrinsic and extrinsic pathways found in humans as well as platelet-specific factors [9]. Evidence has also been presented for the presence of $\mathrm{vWF}$ and its conserved interaction with GPIb $\alpha$ receptor, found on human platelets [10]. Previously, by means of immunostaining, GPIb $\alpha$ was shown to be present on zebrafish thrombocytes, which are involved in forming vascular occlusion upon injury, similarly to human platelets [11]; this further solidifies that zebrafish make an appropriate model for the investigation of vWF and vWD.

In addition to retaining proteins and pathways involved in the clotting process found in humans, zebrafish also provide the advantage of transparent eggs, embryos, and larvae throughout development. This transparency enables investigators to observe development as well as formation of vasculature [10]. The convenience of this model being transparent throughout development, coupled with a variety of genetic and screening tools, provides rapid investigation of dysfunctional proteins involved in the clotting process, disease, and development[11;12] . In this paper, we will provide evidence that vWF function is conserved and aids in the clotting process in zebrafish, just as in humans; and therefore, zebrafish should make a useful model for the study of cell biology of vWF function in vivo.

\section{Materials and Methods}

\section{Zebrafish aquaculture}

The following methods of zebrafish aquaculture were conducted similarly to those previously described [13]. Briefly, adult zebrafish, larvae, and embryos were kept at $28^{\circ} \mathrm{C}$ in deionized water, supplemented with instant ocean, in a circulating water system. Embryos were collected as previously described. 


\section{RT-PCR using Zebrafish Thrombocytes and Whole Larvae and PCR using Zebrafish Genomic DNA}

Thrombocytes were collected from adult zebrafish blood by individually suctioning thrombocytes under the microscope using a microinjection needle. 500 thrombocytes were used for isolating RNA using Absolutely RNA miniprep kit (Stratagene, Inc.; Santa Clara, CA). Total RNA from whole larvae was prepared using the above kit, then used for RT-PCR amplification of vWF mRNA with the following primers: Forward primers: 5'-

TGAGTGGAGATATAACACCTGTGC-3' (F1), 5'CAGTAACTGGTTTAACCTCCACACT-3' (F2), 5'-CTGTTGACGGCAAGTGCTAA-3' (F3), 5'-GAAGCTTTGAGCATTACTGACTACC-3' (F4), and 5'CACAGAGTCCTCCAACTGACG-3' (F5). Reverse primers: 5'TCATCCATGAATGCGACATC-3' (R1), 5'-GAGGTCAGAAGGGTCATCCA-3' (R2), 5'ATGTTTTCAAGTCCTCAAACTG-3' (R3), and 5'GTTTTCACAAATGTTTTCAAGTCCT-3' (R4) (Biosynthesis; Lewisville, TX). F1 is located in the exon corresponding to human exon 26. F2, F3, F4, F5, R1 and R2 are located in the exon corresponding to human exon 28 . R3 and R4 are located in the exon corresponding to human exon 29. The following primers were used for mRNA amplification of EF1- $\alpha$ : forward primer 5'-CGGTGACAACATGCTGGAGG-3' and reverse primer 5'ACCAGTCTCCACACGACCCA-3' were used. Genomic DNA from adult zebrafish was prepared using the Wizard Genomic DNA Purification Kit (Promega; Madison, WI) and was amplified by PCR using two independent primer sets F5R3 and F1R1.

\section{Immunostaining of Whole Larvae}

Whole larvae were fixed in $4 \%$ paraformaldehyde for 6 hours at $4^{\circ} \mathrm{C}$, then washed with 0.1 $\mathrm{M}$ phosphate buffer ( $\mathrm{pH}$ of 7.3) for 5 minutes The larvae were then washed in distilled water for 5 minutes, incubated at $-20^{\circ} \mathrm{C}$ for 7 minutes in acetone, and washed in distilled water for 5 minutes followed by a 5 minute wash in $0.1 \mathrm{M}$ phosphate buffer ( $\mathrm{pH}$ of 7.3).

Subsequently, these larvae were blocked in $2 \%$ goat serum in PBS with $3 \%$ BSA and $1 \%$ DMSO for 1 hour. After blocking, larvae were incubated overnight at $4^{\circ} \mathrm{C}$ in a solution of $1 \%$ DMSO containing either anti-human vWF antibody (vWF-Ab) $8 \mathrm{mg} / \mathrm{ml}$ at a 1:200 dilution (Sigma; St Louis, MI) or control purified rabbit IgG (primary antibody) from nonImmune Sera $10 \mathrm{mg} / \mathrm{ml}$ at a 1:200 dilution (Affinity Biologicals; Ancaster, ON, Canada). After incubation, larvae were rinsed with a solution containing PBS with 3\% BSA and 1\% DMSO for 2 hours with a change to fresh solution every 30 minutes. For visualization, larvae were incubated for 4 hours at $20^{\circ} \mathrm{C}$ in PBS with 3\% BSA and $1 \%$ DMSO with FITC conjugated anti-rabbit IgG (secondary antibody) $2 \mathrm{mg} / \mathrm{ml}$ at a dilution of 1:200 (Jackson Immuno Research; West Grove, PA)

\section{Immunostaining of Thrombocytes}

A blood smear was made using whole blood from adult zebrafish and allowed to dry for 10 minutes. The slide was immersed in $70 \%$ cold ethanol for 10 minutes. Then, the slides were rinsed three times in phosphate buffered saline (PBS) and incubated in vWF-Ab diluted 20 fold in PBS in a total volume of $60 \mu \mathrm{l}$, which was used to cover the blood smear under a coverslip and incubated for 2 hours. After incubation, the slides were rinsed as described above and then incubated with FITC conjugated anti-rabbit IgG (Jackson Immuno Research; West Grove, PA) and diluted 20 times in 1xPBS for 1 hour. Once the second incubation was complete the slides were rinsed with 1xPBS three times; then, the slides were subjected to one final rinse in double distilled water. 


\section{Morpholino Injections}

Embryos were injected with $3 \mathrm{nl}$ of $1 \mathrm{mM}$ antisense MO for vWF, 5'-

ACTGTAGTGTTGATTCTGACCTGAA-3' (Gene Tools; Philomath, OR) for the exonintron boundary of the exon homologous to exon 28 in humans (which encodes for the binding site, in vWF, to GpIb $\alpha$ ), at the one to four cell stages using the picospritzer III (Parker Precision Fluidics; Hollis, NH) [14]. A standard control MO 5'-

CCTCTTACCTCAGTTACAATTTATA-3' was also injected into embryos at the one to four cell stages. Adult zebrafish were injected intravenously with either $5 \mu \mathrm{l}$ of $0.5 \mathrm{mM} v \mathrm{vWF}$ Vivo-Morpholino (VMO), or control VMO designed with the same sequence as the vWF MO, or control MO, respectively (Gene Tools; Philomath, OR) as previously described[15].

Imaging

Images of bleeding larvae, thrombocytes and blood vessels were recorded using either a Nikon Optiphot microscope or Nikon 80i eclipse microscope equipped with NIS Elements AR 2.30 software. TTO was recorded using a Nikon Optiphot microscope as described previously [12].

\section{Injection of Vivo-Morpholino and Agglutination Assay}

Blood was collected from injected fish and used in the ristocetin mediated thrombocyte agglutination assay. The assay was performed as previously described [9]. In order to induce the secretion of vWF, we used $1.5 \mathrm{mg} / \mathrm{ml}$ Stimate (Desmopressin acetate) nasal spray (gift from Shelly Crary, UT Southwestern Medical School) by first spraying it into an Eppendorf centrifuge tube and then diluting the Stimate five fold with distilled water. After the dilution, $5 \mu \mathrm{l}$ of the diluted Stimate was placed on the gills and the fish were returned to water for 30 minutes, after which time blood was collected and the above agglutination assay was performed.

\section{Effect of Morpholino on Time to Occlusion (TTO) and Stimate Effect on TTO}

TTO was measured in seconds on 5-6 day post fertilization (dpf) larvae generated after injection with the $\mathrm{MO}$ at the one to four cell stages by induction of an injury to the artery, using a nitrogen laser light pumped through coumarin 440 dye using a MicroPoint Laser System (Photonic Instruments Inc.; St. Charles, IL) connected to a Nikon Optiphot microscope, after immobilization in $0.8 \%$ agarose by methods previously established [12]. Larvae at 5-6 dpf were placed in Stimate diluted eight fold in distilled water for $15 \mathrm{~min}$., and TTO was measured after placing larvae in agarose as described above.

\section{Analysis of Genome Databases}

ENSEMBL and NCBI BLAST programs were used to identify gene, synteny as well as for alignment of two sequences. For alignment of multiple sequences Biology Workbench 3.2 available through San Diego Super Computer Center was used.

\section{Results}

In our earlier work, we have shown the presence of vWF receptor on thrombocytes in zebrafish. To check whether the vWF gene exists in zebrafish, we analyzed the zebrafish genome database and found that the vWF gene is located on zebrafish chromosome 18, whereas the human vWF gene is situated on human chromosome 12 .

Because synteny of CD9 and vWF is conserved from humans to zebrafish, the proposed $\mathrm{vWF}$ gene present on zebrafish chromosome 18 is the correct one despite the presence of other vWF like genes in the database. However, exon 28, which is conserved in all 
mammalian species, was split into two exons in zebrafish according to the ENSEBML database (Figure 1). The homologies of protein encoded by these two exons appear to have $31 \%$ identity and the $54 \%$ positives; the entire vWF protein showed $46 \%$ identity and $63 \%$ positives. To test whether exon 28 splitting into two exons was unique to zebrafish or whether other fish species have similarly split exons, we analyzed other fish genomes and found that exon 28 is split into two exons in Fugu and Tetraodon but at a different upstream location compared to the region of the split found in zebrafish. In Stickleback and Medaka, exon 28 is split into three portions encompassing the regions of the split found in Fugu and Tetraodon as well as the region of the split found in zebrafish. (Figure 1).

To test for the presence of vWF in zebrafish, we prepared total RNA from whole larvae and adult fish; we then performed RT-PCR using F3 and R2 primers located in the sequence corresponding to human exon 28 (Figure 2). We found a RT-PCR product of $885 \mathrm{bp}$ in size, which was sequenced (data not shown). The sequences confirmed that the amplified product was indeed from vWF mRNA, indicating the presence of vWF transcript in zebrafish. However, instead of lacking the intron sequence predicted by ENSEMBL database it contained this 'intron' sequence. To eliminate the possibility of unspliced product and/or genomic DNA contamination we designed a different set of primers, F4 and R4, located in regions corresponding to human exon 28 and 29, such that F4 is situated upstream from the predicted intron. The RT-PCR yielded a product of $881 \mathrm{bp}$ and, upon sequencing, again demonstrated the presence of the 'intron' sequence. We also designed additional primer sets F2 and R4 as well as F3 and R3 and performed RT-PCRs. We found $1477 \mathrm{bp}$ and $1071 \mathrm{bp}$ RT-PCR products, respectively. These confirmed the inclusion of the predicted intron. These results suggested that the predicted intron is part of vWF mRNA. We also tested whether the reading frame is in line with the continuous translation, and found that there are no termination codons, and the exon 28 sequence translates without any interruption when the predicted intron is included. To check for the presence of introns, we amplified the zebrafish genomic DNA with two independent primer sets F5R3 and F1R1. We found F5R3 the F1R1 primer sets yielded 2501 and 2058 bp bands, respectively. These sizes were consistent with the sizes of the zebrafish introns predicted by the ENSEMBL database.

Since the endothelium of vasculature has been shown to have vWF, we tested for its presence in endothelial cells by using polyclonal antibodies against human vWF and performed whole mount antibody staining using FITC conjugated second antibody. We found the antibody staining in the arteries and veins, as well as intersegmental vessels (Figure 3). These results suggested the presence of vWF in endothelial cells.

Since magakaryocytes have been shown to have vWF transcripts, we hypothesized that thrombocytes, which have transcriptional machinery similar to megakaryocytes, should have vWF transcripts. We tested this hypothesis by preparing RNA from thrombocytes, individually pipetted from zebrafish blood under the microscope, and then performed RTPCR on this RNA using primers F3 and R2. We found the resulting PCR product was 885 bp; this was identical to the product size we found from amplifying the total RNA from the whole larva or adult. Using a different set of primers, F5 and R3, we obtained a 332 bp RTproduct which is the expected size. We also tested the presence of vWF in thrombocytes by immunostaining using anti-human vWF (vWF-Ab) antibody. Thrombocytes showed positive staining in contrast to other blood cells present within the blood smear, confirming the presence of vWF in thrombocytes (Figure 4).

To examine whether vWF has a role in hemostasis, and also to test whether the lack of vWF will lead to bleeding in zebrafish similar to what has been observed in humans and mice, we injected vWF-specific MO designed for exon 27 into one to four cell stage embryos and observed for any visible bleeding. Approximately 50\% of the larvae of 5-6 dpf exhibited 
spontaneous bleeding in head and yolk areas, whereas those injected with the control did not exhibit any bleeding (Figure 5). The larvae that showed minimal bleeding with active circulation were chosen and were subjected to laser induced time to occlusion arterial thrombosis assay. We found the bleeding larvae had an increased TTO that was greater than 120 seconds, whereas the larvae resulting from control morpholino injections showed a normal TTO of 60 seconds (Figure 6). These results showed that vWF plays a role in hemostatic function in zebrafish. To test whether Stimate increases the release of vWF thereby shortening the TTO, we sprayed the $6 \mathrm{dpf}$ larvae with Stimate and measured TTO in the artery, and found that the TTO was shortened (12 seconds) compared to controls (60 seconds) (Figure 6). These results are consistent with the notion that Stimate induces vWF release.

To confirm whether the phenotype was the result of improper splicing due to MO injections, larvae generated from embryos that received $\mathrm{MO}$ were collected and then used for isolating RNA. Using the combination of F1 and R3, we performed RT-PCRs to check whether we would obtain an alternatively spliced $327 \mathrm{bp}$ along with an unspliced $1754 \mathrm{bp}$ product. In this experiment, we obtained a 1754 bp product along with several non specific bands but never could obtain a $327 \mathrm{bp}$ product (data not shown). Other primers designed from the exon 27 also yielded similar results. Furthermore, primers F2 and R4, which should have yielded both 1477 and 811 bp bands, also did not yield an 811 bp band, whereas a 1477 bp band was present. In addition, compared to the control RNA, the $1477 \mathrm{bp}$ band was lighter in those treated with vWF VMO. Therefore, we suspected the mRNA may be unstable due to the loss of exon 28 explaining the loss or reduction in amplification of bands using F5 and R3 primers, in which case amplification in larvae which developed after vWF Mo injections should not yield a band or instead yield a band with reduced intensity; however, EF1- $\alpha$ primers should yield a band. As predicted, we found that in some larvae produced after vWF MO injections, the amplification product disappeared whereas in some cases the product was lighter compared to controls. However, all larvae produced after control MO injections resulted in amplification of vWF. In both experiments, EF-1 $\alpha$ control bands amplified and served as an internal control. We then quantified the ratios of densities for the vWF band and EF1- $\alpha$ band in both larvae produced after vWF MO and control MO injections. We found larvae resulting from control $\mathrm{MO}$ injections had significantly greater levels of $\mathrm{vWF}$ mRNA compared to the larvae produced after vWF MO injections.

To gain further confirmation of whether vWF levels are reduced in plasma due to MO targeting of vWFunspliced mRNA, blood from adult zebrafish injected with vWF VMO was used in the ristocetin mediated thrombocyte agglutination assay. Adult zebrafish injected with control VMO resulted in an agglutination time of 15 minutes, whereas the agglutination time for adult zebrafish blood injected with vWF MO was 45 minutes (Figure 8). These results indicated that the vWF levels are lower in plasma, confirming the larval results.

To test whether Stimate enhances the agglutination of thrombocytes induced by ristocetin, blood from Stimate treated fish were used in the above assay. We found the agglutination of blood from fish treated with Stimate was almost instantaneous compared to the 15 minute agglutination of control zebrafish blood (Figure 8). These results are consistent with the TTO results from larvae treated with Stimate as mentioned above.

Even though we have previously shown, by immunostaining, that GP1b $\alpha$ exists on the thrombocyte surface, we have not shown the presence of genes for GP1b using molecular methods. Therefore, we searched the ENSEMBL database and found the GP1b $\beta$ (accession \#NP_001138284) proteins, which are part of the complex of GP1b $\alpha$ Furthermore, we also found that the Cysteine122 of GP1b $\beta$ which forms disulphide bridge with GP1b $\alpha$ is conserved (Figure 9). 


\section{Discussion}

This paper demonstrated for the first time that vWF is present in the endothelial cells of zebrafish. The fact that we found antibody staining in the arteries and veins, as well as intersegmental vessels, serves as evidence that vWF is made in all endothelial cells irrespective of the vessel type. In mammals, it has also been shown that all endothelial cells make vWF [16]. At present, we do not know whether vWF is stored in the baso-lateral area of the endothelial cells, although the endothelial cells of the fish have been shown to have electron dense organelles that may represent Weibel Palade bodies [17].

It is also interesting to note that thrombocytes also have vWF transcripts, suggesting the synthesis of vWF in these cells. Since we have shown previously that the transcription factors controlling megakaryocytes are well conserved in zebrafish thrombocytes, this suggests that thrombocytes do resemble megakaryocytes in this respect; the synthesis of vWF again adds credence to our earlier observations [18].

Analysis of the zebrafish genome provided us the vWF gene sequence. It is noteworthy that exon 28, which has been shown to be split by the ENSEMBL database, is not split as indicated by sequencing results confirming the presumed intron does exist in the RT-PCR products. If it were to be an unspliced product, we shoud have obtained the correctly spliced product in other reactions. Therefore, the intron suggested by the database does not exist and the $\mathrm{vWF}$ protein carries the additional peptide sequence. Interestingly, the binding site for GP1b $\alpha$ is located in the amino-terminal region of the protein coded by exon 28 and this region also contains two of the three A domains of vWF. Thus, the additional peptide sequence appears to separate these two domains. It is noteworthy that the human mutations are clustered in two regions of exon 28 and the separation of these two domains by a peptide sequence is not affecting the vWF activity in zebrafish. However, the loss of these domains would result in a vWD-like phenotype. Indeed, in this paper, we observed that the disruption of splicing of vWF RNA will lead to spontaneous bleeding in zebrafish due to the loss of exon 28. This observation is remarkably similar to the bleeding phenotype observed in humans with von Willebrand Disease. This result along with the TTO assays provided the functional evidence that $\mathrm{vWF}$ is important in zebrafish hemostasis.

Although we observed the bleeding phenotype, we could not obtain the alternatively spliced products in two separate experiments that amplified vWF RNA using two independent primer sets. Such results suggest that the alternatively spliced mRNA appears to be unstable under our experimental conditions. The clear absence of a vWF-specific band when EF1- $\alpha$ has been amplified supports this interpretation. Also, the functional evaluation of thrombocyte agglutination in vitro, using zebrafish blood after vWF VMO injection, with ristocetin strongly supports the reduction of vWF in plasma. Furthermore, our finding that GP1b $\beta$ gene exists and has a conserved Cysteine122 that forms a disulphide bridge with GP1b $\alpha$ lends support to our earlier finding that the receptors for vWF are conserved in fish [19].

In conclusion, we demonstrated the presence of vWF in both endothelium and thrombocytes, and showed the bleeding phenotype due to vWF MO performed along with other lines of evidence, such as: a lack of alternatively spliced vWF mRNA, a delay in larval arterial thrombosis, a delayed ristocetin-mediated agglutination in zebrafish adult blood. Delayed agglutination time indicated that a reduction in vWF levels due to vWF MO, implies the presence of sequences that are receptors for vWF on thrombocytes. Thus, we propose that the zebrafish model will prove to be an asset in the study of vWF function in vivo.

Blood Cells Mol Dis. Author manuscript; available in PMC 2011 December 15. 


\section{References}

1. Sadler JE. Biochemistry and genetics of von Willebrand factor. Annu Rev Biochem. 1998; 67:395424. [PubMed: 9759493]

2. Loscalzo, J.; Schafer, AI. Thromosis and Hemorrhage. Williams and Wilkins; Baltimore: 1998.

3. Ruggeri ZM. The role of von Willebrand factor in thrombus formation. Thromb Res. 2007; 120(Suppl 1):S5-9. [PubMed: 17493665]

4. Mannucci PM. Treatment of von Willebrand's Disease. N Engl J Med. 2004; 351:683-94. [PubMed: 15306670]

5. Brinkhous KM, Reddick RL, Read MS, Nichols TC, Bellinger DA, Griggs TR. von Willebrand factor and animal models: contributions to gene therapy, thrombotic thrombocytopenic purpura, and coronary artery thrombosis. Mayo Clin Proc. 1991; 66:733-42. [PubMed: 2072761]

6. Denis CV, Wagner DD. Insights from von Willebrand disease animal models. Cell Mol Life Sci. 1999; 56:977-90. [PubMed: 11212329]

7. Jagadeeswaran P, Gregory M, Day K, Cykowski M, Thattaliyath B. Zebrafish: a genetic model for hemostasis and thrombosis. J Thromb Haemost. 2005; 3:46-53. [PubMed: 15634265]

8. Jagadeeswaran P, Sheehan JP. Analysis of blood coagulation in the zebrafish. Blood Cells Mol Dis. 1999; 25:239-49. [PubMed: 10575549]

9. Jagadeeswaran P, Sheehan JP, Craig FE, Troyer D. Identification and characterization of zebrafish thrombocytes. Br J Haematol. 1999; 107:731-8. [PubMed: 10606877]

10. Isogai S, Horiguchi M, Weinstein BM. The vascular anatomy of the developing zebrafish: an atlas of embryonic and early larval development. Dev Biol. 2001; 230:278-301. [PubMed: 11161578]

11. Summerton J. Morpholino antisense oligomers: the case for an RNase H-independent structural type. Biochim Biophys Acta. 1999; 1489:141-58. [PubMed: 10807004]

12. Gregory M, Hanumanthaiah R, Jagadeeswaran P. Genetic analysis of hemostasis and thrombosis using vascular occlusion. Blood Cells Mol Dis. 2002; 29:286-95. [PubMed: 12547218]

13. Jagadeeswaran P, Liu YC. Developmental expression of thrombin in zebrafish embryos: a novel model to study hemostasis. Blood Cells Mol Dis. 1997; 23:147-56. [PubMed: 9236153]

14. Day K, Krishnegowda N, Jagadeeswaran P. Knockdown of prothrombin in zebrafish. Blood Cells Mol Dis. 2004; 32:191-8. [PubMed: 14757435]

15. Kim S, Radhakrishnan UP, Rajpurohit SK, Kulkarni V, Jagadeeswaran P. Vivo-Morpholino knockdown of alphaIIb: A novel approach to inhibit thrombocyte function in adult zebrafish. Blood Cells Mol Dis. 44:169-74. [PubMed: 20045356]

16. Giddings JC, Banning AP, Ralis H, Lewis MJ. Redistribution of von Willebrand factor in porcine carotid arteries after balloon angioplasty. Arterioscler Thromb Vasc Biol. 1997; 17:1872-8. [PubMed: 9351348]

17. Ferri S, Sesso A. Tubulated bodies in teleost (Pimelodus maculatus) endothelial cells. Arch Anat Microsc Morphol Exp. 1983; 72:19-22. [PubMed: 6639042]

18. Jagadeeswaran P, Lin S, Weinstein B, Hutson A, Kim S. Loss of GATA1 and gain of FLI1 expression during thrombocyte maturation. Blood Cells Mol Dis. 2010; 44:175-80. [PubMed: 20110178]

19. Luo SZ, Mo X, Afshar-Kharghan V, Srinivasan S, Lopez JA, Li R. Glycoprotein Ibalpha forms disulfide bonds with 2 glycoprotein Ibbeta subunits in the resting platelet. Blood. 2007; 109:6039. [PubMed: 17008541] 

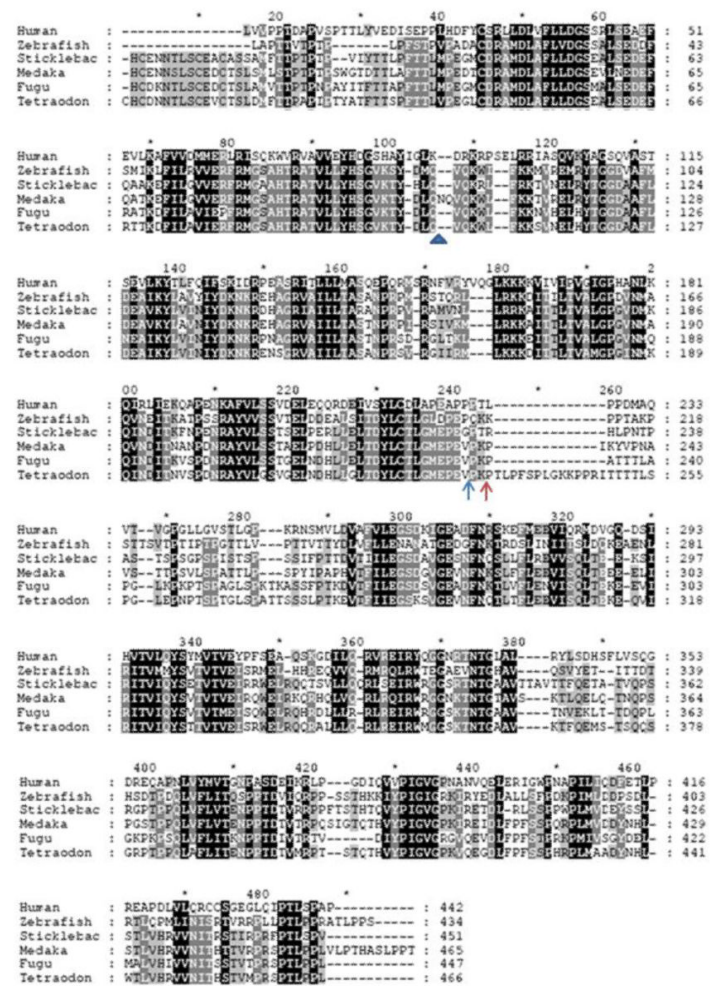

Figure 1.

Amino acid alignment of vWF protein sequence corresponding to human exon 28. Arrow head points to the region where exon 28 of humans is split in all fishes with the exception of zebrafish. Blue arrow points the regions where exon 28 in humans is split in Stickleback and Medaka. Red arrow points the regions where exon 28 in humans is split in Zebrafish (ENSEMBL database). 

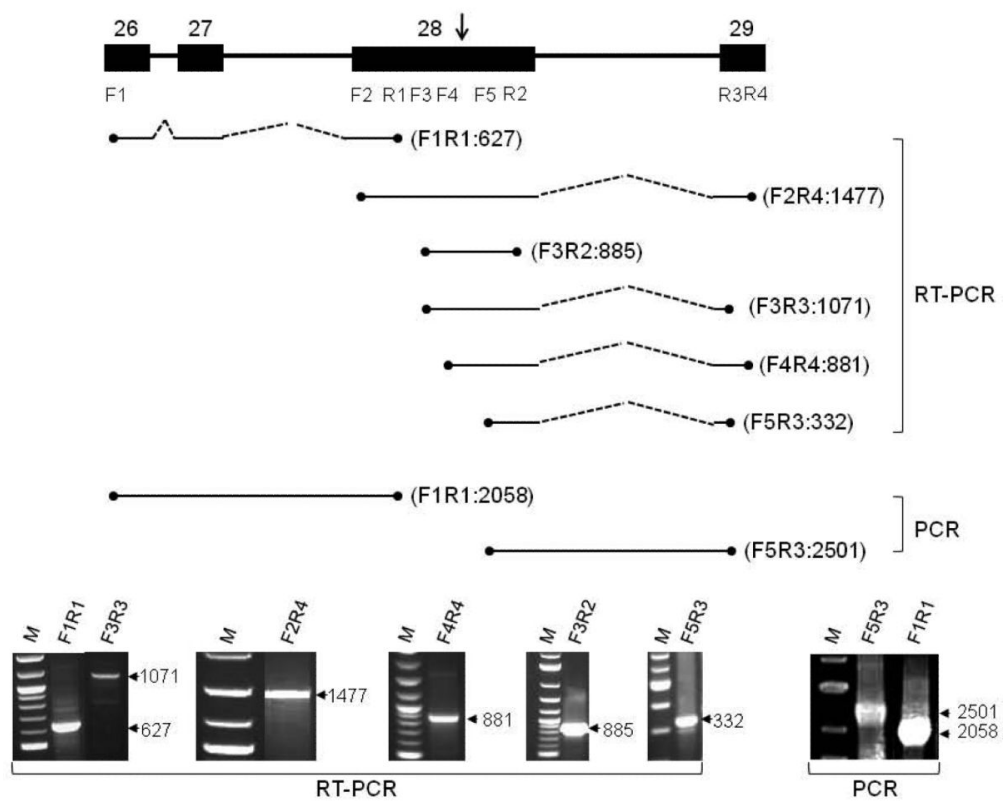

Figure 2.

Schematic diagram of the RT-PCR amplified cDNA products from exon 26 through exon 29 using RNA collected from zebrafish larvae and PCR products amplified using zebrafish genomic DNA, respectively. Exons are shown as black boxes with the numbering on top corresponding to the human exon numbering. The arrow points to the location of the 'intron' according to ENSEMBL database. Forward primers are numbered F1 to F5 whereas the reverse primers are numbered R1 to R4 and are shown beneath the exons. RT-PCR product sizes are shown by lines flanked by solid circles (not drawn to scale). Solid circles represent roughly the primers locations within the exons; dashed lines show the introns removed by splicing. The forward and reverse primer combination is shown in parenthesis followed by the size of the product in base pairs. The agarose gel photographs show the amplified products corresponding to those shown in the schematic diagram. Lanes of the amplified products are marked with the combination of primers; M shows the DNA size markers. Sizes of the amplified products in base pairs are shown by arrows. The cDNA products generated by RT-PCR and the genomic products generated by PCR are marked separately. 


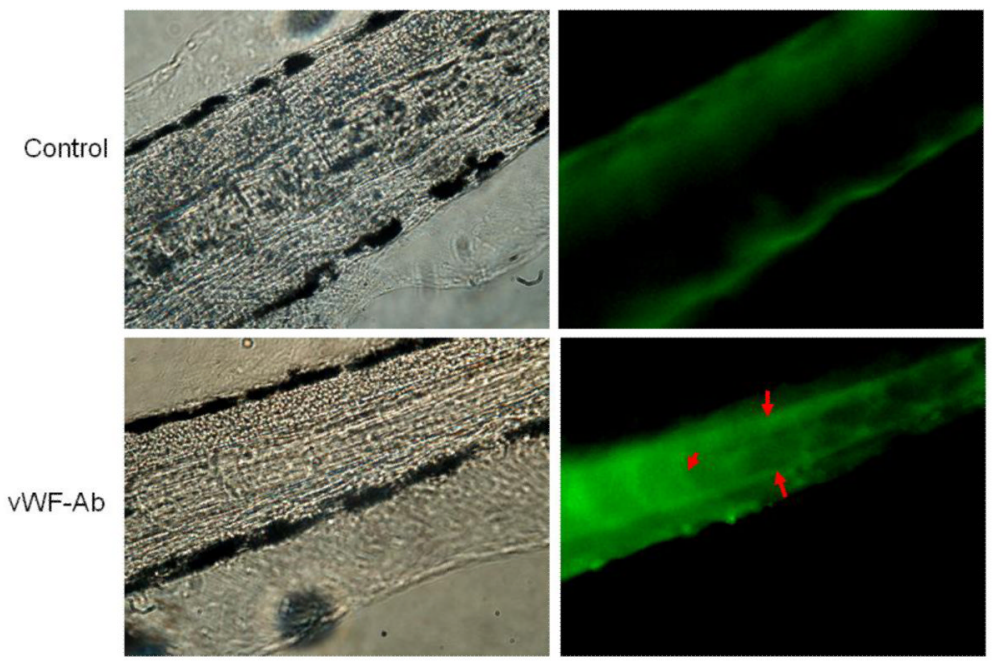

Figure 3.

Immunostaining of blood vessels. Human von Willebrand factor antibody (vWF-Ab) and rabbit IgG (Control) were used as primary antibodies followed by FITC conjugated secondary antibody in immunostaining. Left and right panels show the brightfield and fluorescence images respectively. Large arrows show caudal artery (upper region) and caudal vein (lower region) whereas the small arrow shows the intersegmental vessels. 


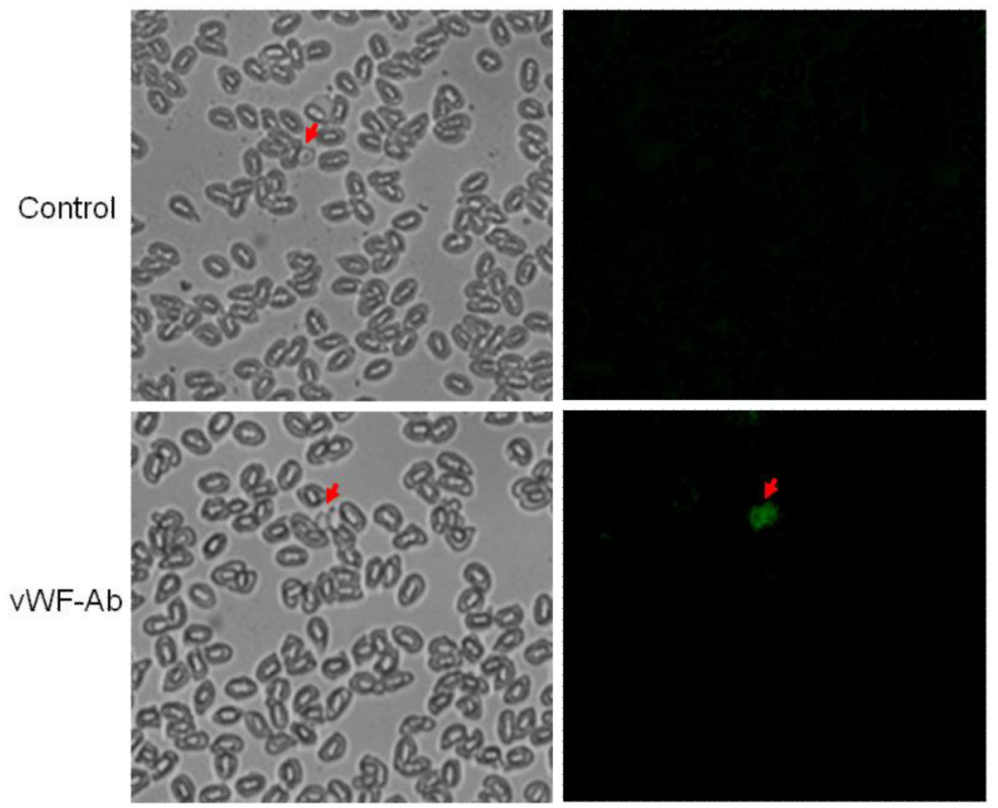

Figure 4.

Immunostaining of thrombocytes. Human von Willebrand factor antibody (vWF-Ab) and rabbit IgG (Control) were used as primary antibodies followed by FITC conjugated secondary antibody. Left and right panels show the brightfield and fluorescent images respectively. Arrows point to the thrombocytes, all other surrounding cells are red cells. 


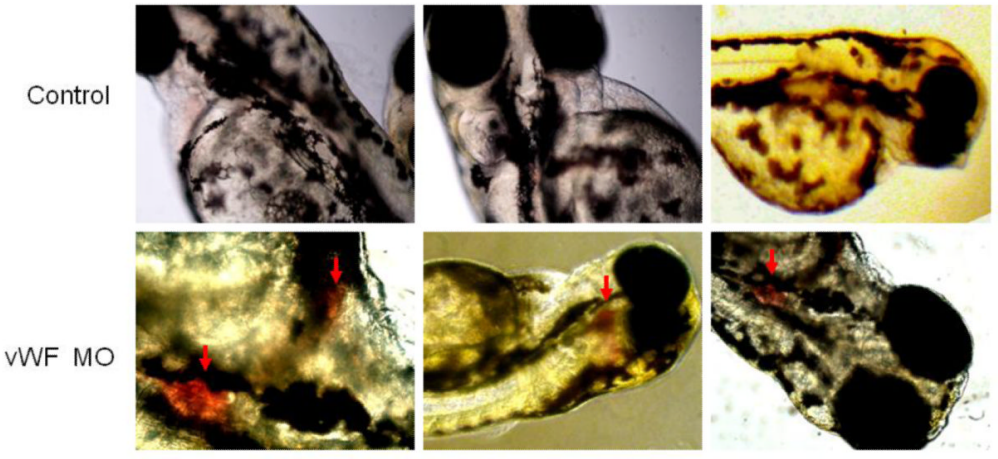

Figure 5.

Larvae generated after injecting vWF MO (bottom panels) and control MO (top panels) into the 1-4 cell-stages of embryos. Arrows indicate the location of bleeding in the head and yolk region. 

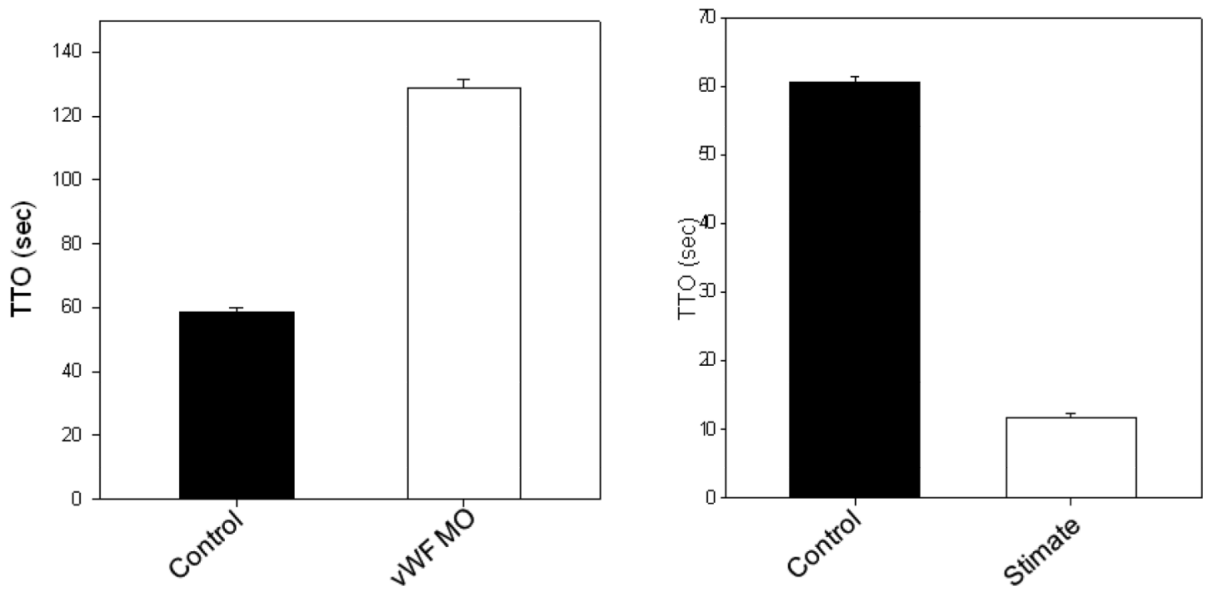

Figure 6.

TTO in 6 dpf zebrafish larvae. TTO using larvae generated after injecting vWF MO and control MO into 1-4 cell-stages of embryos, $n=14, p=<0.001$ (left panel). TTO using larvae treated with Stimate and untreated controls, $n=7, p=<0.001$ (right panel). 

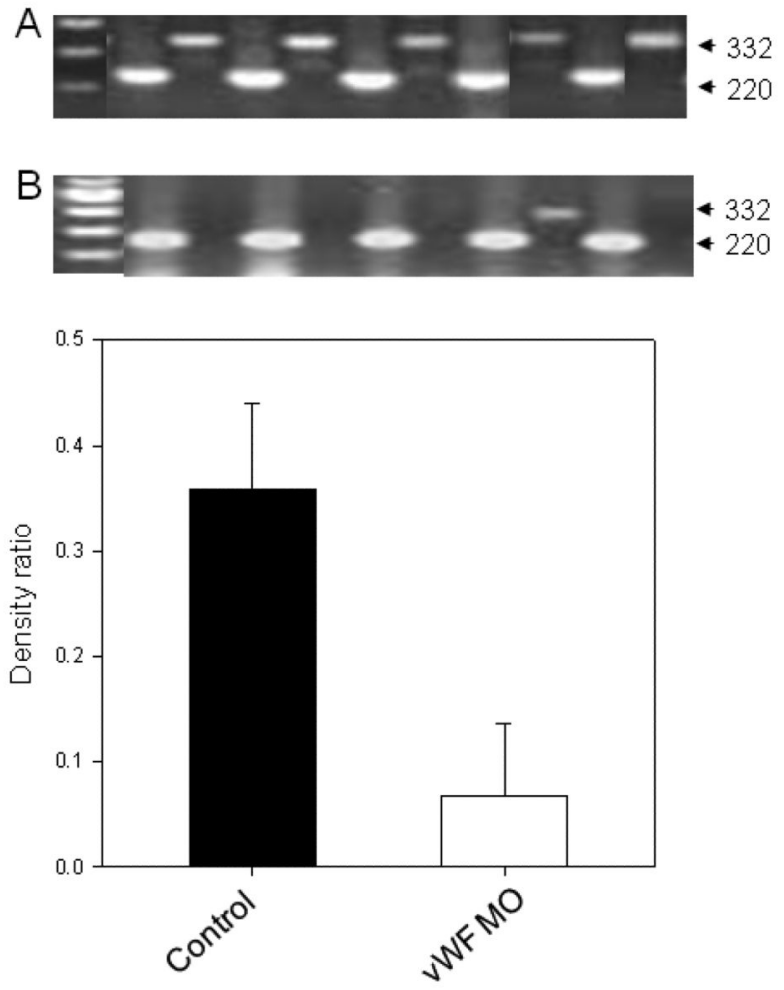

Figure 7.

Analysis of splicing of vWF mRNA after treating the embryos with vWF MO. A and B show representative RT-PCR products from RNA collected from 24-72 hrs post fertilization embryos injected with control MO and vWF MO at 1-4 cell-stages of embryos respectively. Arrows show the size of the RT-PCR products in base pairs (332 for vWF and 220 for EF1$\alpha$ ) on agarose gel photographs. Left most lanes show the DNA size markers. The bar graph shows the 220 bp band/332 bp band densities, $n=30, p=<0.001$. 

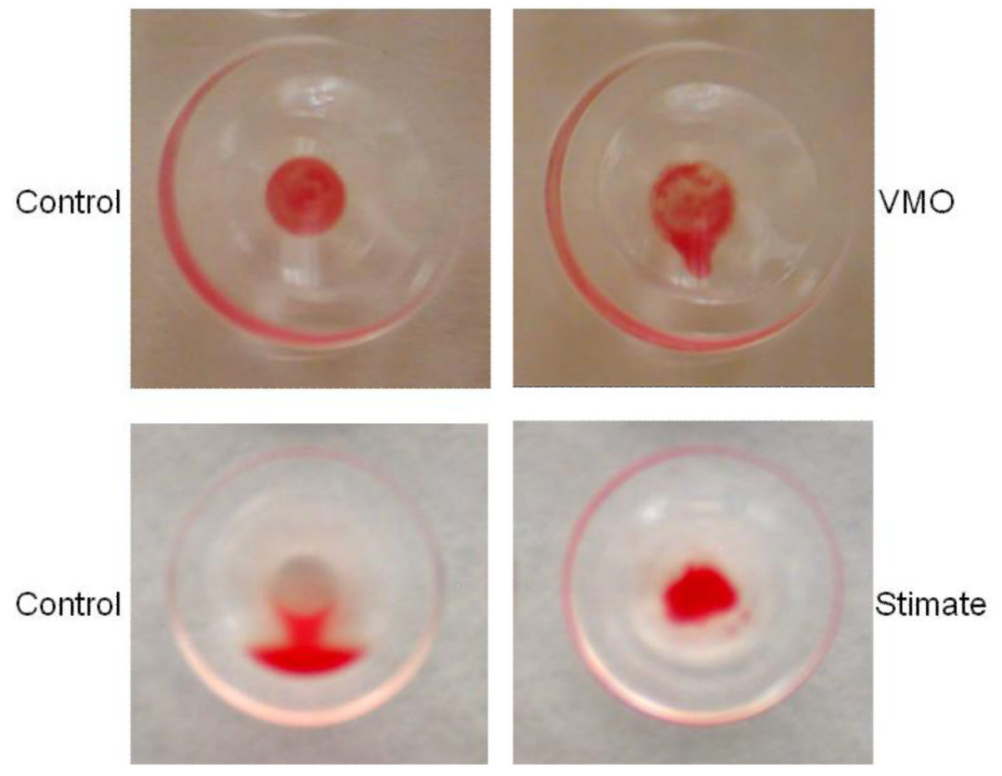

Figure 8.

Ristocetin mediated thrombocyte agglutination assay. Top panels show thrombocyte aggregation using whole blood from control MO and vWF MO injected adults. Bottom panels show thrombocyte aggregation using whole blood from Stimate treated and untreated control adult zebrafish. 


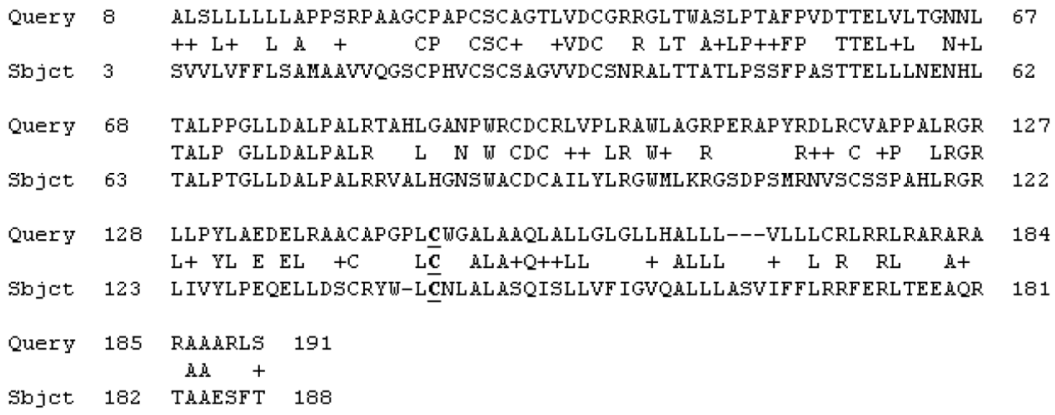

Figure 9.

Amino acid alignment of human and zebrafish GP1b $\beta$ 